suggest that in the germinating pea, ribonuclease is involved in the translocation of polynucleotide material by bringing about degradation of ribonucleic acid in the cotyledons and re-synthesis in the root and shoot. This mechanism, since it does not involve nucleoside $5^{\prime}$-polyphosphates as intermediates, could operate without interference with the synthesis of nucleotide coenzymes in the growing parts of the plant.

G. R. BARKER

Thelma Dovglas

Department of Chemistry,

The University,

Manchester, 13.

${ }^{1}$ Brown, D. M., and Todd, A. R., J. Chem. Soc., 44 (1952).

Heppel, L. A., Whitfeld, P. R., and Markham, R., Biochem. J., 60, 8 (1955). ${ }^{3}$ Sato-Asano, K., and Egami, F., Biochim. Biophys. Acta, 29, 655

4 Harker, G. R., Montague, D. M., Moss, R. J., and Parsons, M. A., J. Chem. Soc, 3786 (1957).

${ }^{5}$ Reddi, K., Biochim. Biophys. Acta, 33, 164 (1959).

${ }^{3}$ Holden, J., and Pirie, N. W., Biochem. J., 60, 39 (1955).

\section{Mechanism of the Action of Thymidylate Synthetase}

Ir has been postulated ${ }^{1-4}$ that the enzymic synthesis of thymidylic acid from deoxyuridylic acid occurs according to the equations :

$$
\text { Tetrahydrofolate }+\underset{\text { tetrahydrofolate }+\mathrm{H}_{2} \mathrm{O}}{\mathrm{HCHO}} \underset{\mathrm{N}^{5}, \mathrm{~N}^{10} \text {-methylene }}{\rightleftharpoons}
$$

$\mathrm{N}^{5}, \mathrm{~N}^{10}$-Mothylene tetrahydrofolate + deoxyuridylate $\rightarrow$ dihydrofolate + thymidylate

Previous evidence that dihydrofolate is a product has been indirect, since it rested on: (1) balance studies which indicated that tetrahydrofolate is a substrate, and that it assumes the role of catalyst only under conditions where it is regenerated ${ }^{1,3,4}$; (2) isotope experiments in which it was shown that hydrogen isotope from tetrahydrofolate is incor. porated into the methyl group of thymidylate ${ }^{2}$.

We have now obtained direct evidence that dihydrofolate is a product of the reaction by experiments with thymidylate synthetase purified 100-fold from sonic extracts of Streptococcus faecalis $\mathbf{R}$. Dihydrofolic reductase purified 300 -fold from $S$. faecalis R. was used to generate lu-tetrahydrofolate in the reaction mixture and the latter compound then reacted with formaldehyde to give methylene tetrahydrofolate, which produced a characteristic absorption spectrum. In the presence of the purified thymidylate synthetase (activity $10 \mu$ moles of thymidylate synthesized $/ \mathrm{hr}$./mgm. protein), addition of deoxyuridylate caused a spectrum ehange, the rate of which has been used to determine enzyme activity. On completion of the reaction the spectrum was that of dihydrofolate. The product with this spectrum was hydrogenated by reduced triphosphopyridine nucleotide in the presence of the highly specific dihydrofolic reductase and the reduction was inhibited by aminopterin, which is a specific inhibitor of folic and dihydrofolic reductases.

Friedkin and Kormberg postulated ${ }^{5}$ that in the thymidylate synthetase reaction deoxyuridylate and methylene tetrahydrofolate react to form an intermediate in which a methylene bridge links $\mathrm{C}^{5}$ of uracil to $\mathrm{N}^{5}$ (or $\mathrm{N}^{10}$ ) of tetrahydrofolate. During the course of our work on the purification of thymidyl- ate synthetase, none of the many fractionations has resulted in even partial separation of activity into the two different enzyme fractions which would be required to synthesize and cleave such an inte:mediate. When the reaction was followed spectrophotometrically there was no indication of a change in reaction-rate of the kind to be expected if an intermediate accumulated in the reaction mixture. Moreover, two well-defined isosbestic points were observed for the spectrum change, indicating that dihydrofolate was the only new species formed in significant amounts from methylene tetrahydrofolate.

In isotope experiments we have generally used labelled glucose-6-phosphate- ${ }^{3} \mathrm{H}$, glucose-6-phosphate dehydrogenase and triphosphopyridine nucleotide to hydrogenate dihydrofolate in the presence of dihydrofolic reductase to yield lL-tetrahydrofolate- ${ }^{3} \mathrm{H}$. The hydrogen transfers proceeded to completion and the entire product was then allowed to react according to equations (1) and (2). The thymidylate formed was degraded to thymine, which was isolated by paper chromatography. The specific activity of the thymine did not differ significantly from that of the hydrogen attached to carbon-l of the glucose-6-phosphate. The latter was estimated by forming glutamate ${ }^{3} \mathrm{H}$ from the glucose-6-phosphate- ${ }^{3} \mathrm{H}$ in the presence of appropriate enzymes and substrates ${ }^{6}$. When tritiated water and unlabelled reduced triphosphopyridine nucleotide were used in a simila. sequence of reactions the isolated thymine had only 2 per cent of the specific activity of the water. These results are similar to those obtained by Friedkin" with chemically synthesized dlL-tetrahydrofolate, except that in our experiments the transfer of hydrogen occurred without significant dilution, and confirm that one hydrogen of the thymine methyl group is derived from tetrahydrofolate.

This evidence suggests that thymidylate synthetase is a single enzyme and that no intermediate of the type suggested by Friedkin and Kornberg 5 accumulates in significant amounts, although its transitory. formation on the enzyme surface would be consistent with the isotope results and other data.

\section{Barbara M. McDougall*}

R. L. BLAKLEY

Department of Biochemistry,

John Curtin School of Medical Research,

Australian National University, Canberra, A.C.T.

* Australian National University scholar.

'Humphreys, G. K., and Greenberg, D. M., Arch. Biochem. Biophys. 78, 275 (1958).

2 Friedkin, M., Fed. Proc., 18, 230 (1959).

${ }^{3}$ McDougall, B. M., and Blakley, R. L., Biochim. Biophys. Actu, 39, $176(1960)$.

4 McDougall, B. M., and Blakley, R. L., J. Biol. Chem. (in the press). - Friedkin, M., and Kornberg, A., Symp. Chemical Basis of Heredity, Baltimore, 609 (1957).

S Stern, 13. K., and Vennesiand. B., .T. Biol. Chem., 235, 205 (1960)

\section{PHYSIOLOGY}

\section{Derivation of an Equation for the Diffusion Potential}

AN equation for the potential across a membrane arising from the steady-state diffusion of univalent ions was derived by D. Goldman ${ }^{1}$ under the assump. tions of a constant electric field and constant ionic mobilities across the membrane. His equation may be written as $^{2}$ : 